\title{
Prediction of uridine modifications in tRNA sequences
}

Bharat Panwar ${ }^{1,2}$ and Gajendra PS Raghava ${ }^{1 *}$

\begin{abstract}
Background: In past number of methods have been developed for predicting post-translational modifications in proteins. In contrast, limited attempt has been made to understand post-transcriptional modifications. Recently it has been shown that tRNA modifications play direct role in the genome structure and codon usage. This study is an attempt to understand kingdom-wise tRNA modifications particularly uridine modifications (UMs), as majority of modifications are uridine-derived.

Results: A three-steps strategy has been applied to develop an efficient method for the prediction of UMs. In the first step, we developed a common prediction model for all the kingdoms using a dataset from MODOMICS-2008. Support Vector Machine (SVM) based prediction models were developed and evaluated by five-fold cross-validation technique. Different approaches were applied and found that a hybrid approach of binary and structural information achieved highest Area under the curve (AUC) of 0.936. In the second step, we used newly added tRNA sequences (as independent dataset) of MODOMICS-2012 for the kingdom-wise prediction performance evaluation of previously developed (in the first step) common model and achieved performances between the AUC of 0.910 to 0.949 . In the third and last step, we used different datasets from MODOMICS-2012 for the kingdom-wise individual prediction models development and achieved performances between the AUC of 0.915 to 0.987 .

Conclusions: The hybrid approach is efficient not only to predict kingdom-wise modifications but also to classify them into two most prominent UMs: Pseudouridine $(Y)$ and Dihydrouridine (D). A webserver called tRNAmod (http://crdd. osdd.net/raghava/trnamod// has been developed, which predicts UMs from both tRNA sequences and whole genome.
\end{abstract}

Keywords: Uridine modifications, Pseudouridine, Dihydrouridine, 5-methyl-uridine, tRNAmod

\section{Background}

Post-transcriptional modification plays an imperative role in tRNA secondary and tertiary structure formation [1,2], stability [3-6] and ultimately affects tRNA functions $[7,8]$. Sometimes it leads to the alternative folding of tRNAs [1]. It provides structural flexibility to tRNA and rigidifies certain regions to fine-tune the molecule for maximum performance $[3,9]$. It affects the gene expressions [10], translation speed and accuracy [11,12]; enhances the accuracy of codon binding [13] and codon discrimination ability of tRNAs [14]. Modification prevents frame shifting $[15,16]$ that is required for the maintenance of proper translational reading frame $[10,17]$ and enables translocation of the tRNA from $\mathrm{A}$ to $\mathrm{P}$ site

\footnotetext{
* Correspondence: raghava@imtech.res.in

'Bioinformatics Centre, CSIR-Institute of Microbial Technology, Sector 39A, Chandigarh, India

Full list of author information is available at the end of the article
}

[18]. Some modified bases, particularly modifications of anticodon domain help in the amino-acylation reaction of aminoacyl-tRNA synthetases through recognition of cognate tRNAs [19-21]. It is a principal reaction for the precise flow of genetic information into protein sequences [22,23].

The position of modified base in tRNA sequence is also important because modified wobble position 34 expands tRNA ability to read more than one codons [24]. It contributes $30-40 \%$ of all codon recognition depending on the codon usage of an organism [8]. $U_{34}$ is mostly modified $[25,26]$ and is responsible for the majority of wobble based codon recognitions $[7,8,27,28]$. The tRNA modifications are involve in various diseases such as Type 2 diabetes [29-31], Cancer [32-35] and mitochondrial disease [36]. Modification also plays important role in human immunodeficiency virus selection of a specific human tRNA to prime reverse transcription [37]. A recent study showed 
that tRNA modifications play an important role in genome structure and codon usage [38]. Yet, cellular and functional dynamics of tRNA modifications is unexplored and poorly understood due to the absence of large-scale analysis and quantification of modifications. The experimental determination of tRNA modification is also an expensive, tedious and labor-intensive process. Therefore, there is a need to develop an algorithm for the prediction of tRNA modifications.

In this study, an attempt has been made to develop insilico technique for identification of modified bases in tRNA sequence. We retrieved and analyzed modified tRNA from MODOMICS database [39,40] and observed that most of the modifications are uridine-derived. Therefore, we focused our study on the prediction of uridine modifications (UMs) in tRNA. We used various features such as compositions, binary and structural information of tRNA for developing Support Vector Machines (SVMs) based models for identification of modified uridine in tRNA. It was observed that Pseudouridine (Y) and Dihydrouridine (D) were more prevalent modifications in the tRNA sequences. Therefore separate prediction models have been developed for these (D and $\mathrm{Y}$ ) prominent uridine modifications (UMs). It is known that modification varies between different kingdoms; therefore we also developed kingdom-specific prediction models. This sequence based prediction and classification of UMs will help the scientific community to explore tRNA biology. In this era of Next-Generation Sequencing (NGS), tRNAmod tool developed in this study will be useful for the genomewide prediction of tRNA modifications.

\section{Results}

In this study, two different version of MODOMICS database update 2008 [39] and update 2012 [40] have been used. Update 2008 and 2012 of MODOMICS database were containing 218 and 642 tRNA (modified) sequences respectively. In the analysis part, we used all the 642 tRNAs of the 2012 update. We analyzed position-specific base conservation in standard 1-99 (or 0-98) representation using WebLogo [41] and observed that some positions were conserved whereas most of positions have variants (Additional file 1: Figure S1). Thus, determination of position-specific modification in the variable region is a major challenge.

\section{Analysis of all tRNA modifications}

We observed that $\sim 13 \%$ bases were modified. It means that on an average, 10 bases of each tRNA (average 77 nucleotides long) were modified. The nucleotide-compositions of $\mathrm{U}, \mathrm{G}, \mathrm{A}, \mathrm{C}$ and any/other in tRNA sequences were $24.31 \%$, $27.62 \%, 22.5 \%, 25.34 \%$ and $0.22 \%$ respectively. The U-, G-, A- and C-derived modifications were 55.85\%, 19.71\%, $12.28 \%$ and $10.41 \%$ of all tRNA modifications respectively.
Base specific modification rate varies between the different kingdoms but still uridine-derived modifications are most abundant in all the kingdoms (Additional file 1: Figure S2). The $29.27 \%$ of all uridines were modified whereas only $9.09 \%$ of guanines, $6.94 \%$ of adenines and $5.21 \%$ of cytosines were modified. We observed in the kingdom-wise Two Sample Logos (TSLs) of modified and unmodified patterns (sliding windows of 15 lengths) that uridines were also most abundant in the neighboring positions of modified base whereas cytosines and guanines were preferred in the neighboring positions of unmodified bases (Figure 1). Most of modifications were uridine-derived; therefore we selected only these modifications for further study.

\section{Analysis of uridine modifications (UMs)}

There are several modifying enzymes that play important role in the post-transcriptional modifications of tRNA. It is important to investigate the differences between modified $(29.27 \%)$ and unmodified uridines. Therefore, we created kingdom-wise TSLs and observed that significant differences were present between modified and unmodified uridines (Figure 2). The bases of central (8th) position were modified/unmodified uridines. The modified uridines were flanked by guanine ( $5^{\prime}$ end) and uridine (both $5^{\prime}$ and 3 ' end) whereas unmodified uridines preferred cytosine ( $5^{\prime}$ end) and guanine ( $3^{\prime}$ end) as neighbors. Most of modified uridines preferred guanine at 6 th and adenine at 11-12th positions (Figure 2). Although, there were 22 different type of uridine-modifications present but Pseudouridine $(\mathrm{Y})$ was most ( $45 \%$ of all UMs) abundant UMs (Additional file 1: Table S1). Sequence-based conservation of pseudouridine modifications was analyzed and observed that there was very low conservation present (Figure 3). Only some conservation of uridine at $5^{\prime}$ and cytosine at 3 'end was present (especially in bacteria). It is well known because TYC (UUC in the WebLogo) is always present in TSL but pseudouridine is also present at other sites. The Dihydrouridine (D) was second most abundant $(\sim 32 \%$ of all UMs) and only present in the DSL but as WebLogos suggested that there was no sequence-based conservation present for this modification (Figure 3). On the basis of these analyses, we developed prediction models for the all uridine-modification, pseudouridine and dihydrouridine separately.

In this study, we used a three-step strategy to develop an efficient method for the prediction and classification of uridine modifications. In first step, we developed a common prediction method using tRNA-136 dataset of MODOMICS database update 2008 [39]. In the second step, we used newly added tRNA sequences (as independent dataset) of MODOMICS database update 2012 [40] for the kingdom-wise prediction performance evaluation of previously developed (in first step) common model. In the last step, we used tRNA-419 and tRNA-471 


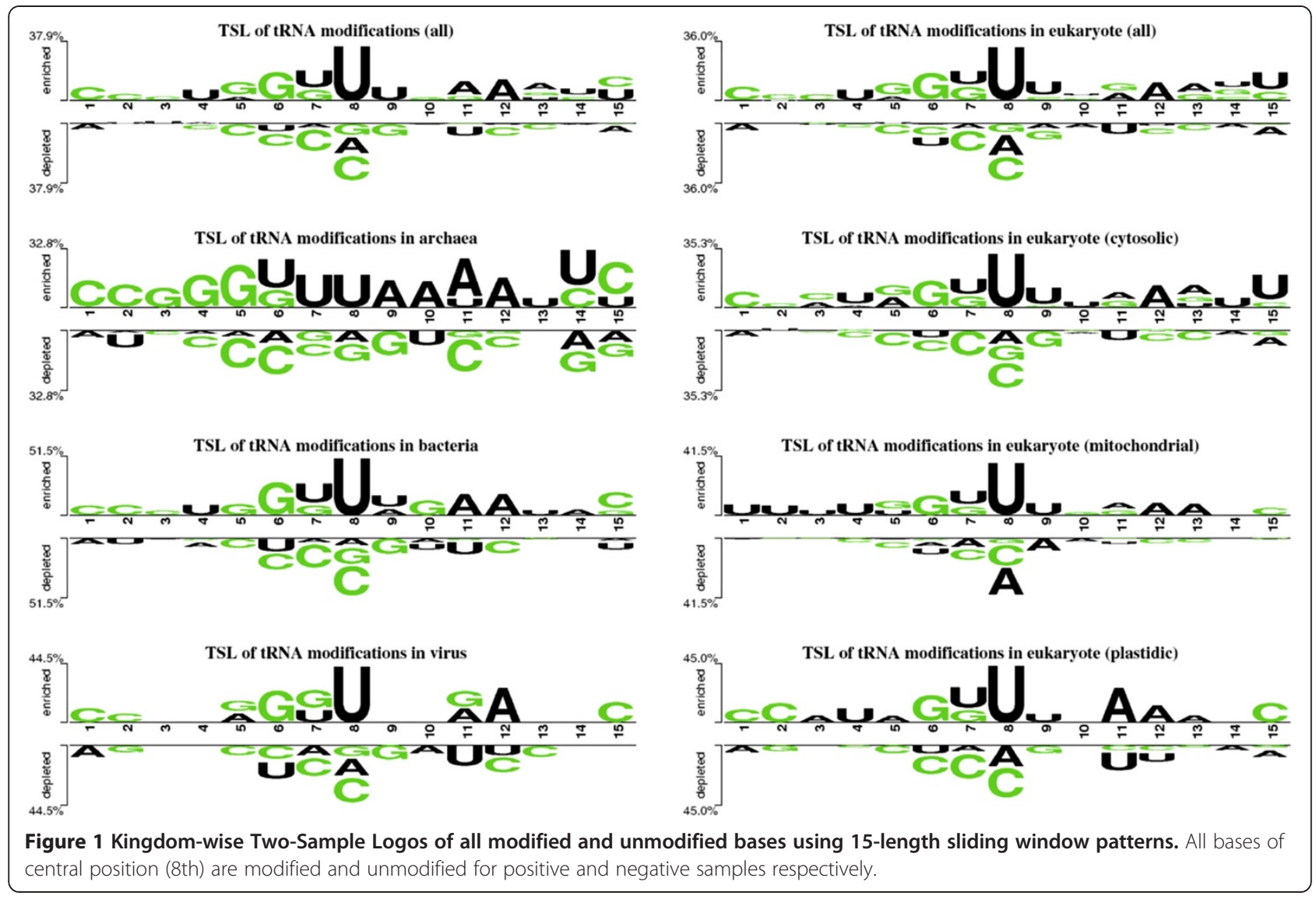

datasets (See details in the Methods section) of MODOMICS database update 2012 for the kingdom-wise prediction model development.

\section{Identification of uridine modifications}

In past, various machine learning based prediction methods have been developed for biological problems and SVM is one of the most powerful and highly used algorithms. First, we generated sliding window of different lengths and created positive and negative patterns. If central nucleotide of window is modified uridine, the whole pattern was used as positive otherwise windows with unmodified uridines at central positions were used as negative patterns (see material and methods section). We optimized window sizes by prediction performances and applied following approaches and developed various SVM-based prediction models. In first step, all models were developed on a non-redundant dataset called 'tRNA-136' (see Methods section), which contain 136 tRNA sequences, where no two sequences have more than $50 \%$ sequence similarity.

\section{Compositions-based approaches}

We developed various SVM based modules for predicting modified uridine in tRNA using mono-, di- and trinucleotide composition (Additional file 1: Figures S3-S5) and optimized window size for achieving best performance in terms of area under curve (AUC). We achieved maximum AUC 0.76, 0.84 and 0.865 for mono-, di- and trinucleotide composition respectively.

\section{Binary approach}

The compositions-based approaches give information of only nucleotide frequencies; it has no information about sequential arrangement of these nucleotides. Therefore, we applied binary approach, which is widely used and is a successful strategy for the nucleotide (or residue) level predictions [42]. First, we generated binary profiles of patterns (BPP) of length 3 to 25 nucleotides. These BPPs were used to develop SVM based methods for predicting modified uridine in tRNA sequences. We computed performance of window length 3 to 25 and achieved maximum MCC 0.72 with accuracy $89.13 \%$ and AUC 0.924 at 17-window length (Additional file 1: Figure S6).

\section{Structure-based approaches}

All nucleotide sequences of tRNAs fold into welldefined cloverleaf like structures. There are loop-specific UMs presents in D-stem loop (DSL), T-stem loop (TSL), Anticodon-stem loop (ASL) and Variable loop (VL). Therefore, this structural information can also be useful for the 


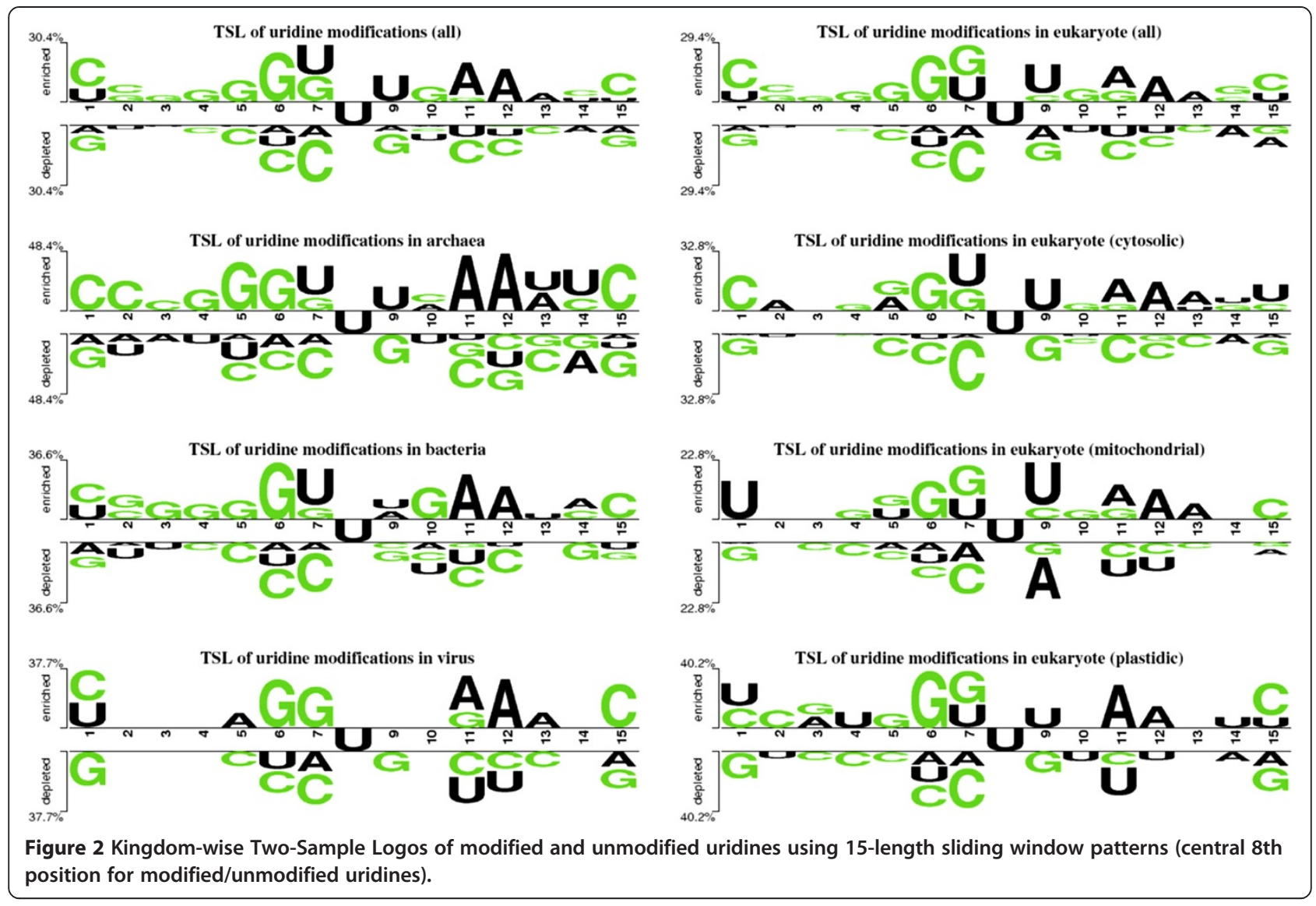

prediction of UMs. We used three different software packages namely RNAfold [43], IPknot [44] and tRNAscan-SE [45] for predicting structure of tRNA. The binary representation of predicted tRNA structure was used for developing SVM based models (see material and methods section). The performance of SVM models were based on the binary representation of structures tRNA, predicted using RNAfold, IPknot and tRNAscan-SE shown in Additional file 1: Figures S7, S8 and S9 respectively. At window length 19, we achieved MCC 0.73 with AUC 0.925 for models based on predicted structures using tRNAscan-SE. One possible reason of better performance of tRNAscan-SE approach is that it was developed specifically for tRNA and it predicted DSL, ASL, VL and TSL boundaries correctly in comparison to RNAfold and IPknot.

\section{Hybrid approach}

As shown in above sections, SVM models based on BPP (window length 17) and on tRNAscan-SE (window length 19) predicted structures performed better than other models. In order to improve performance of our approach, we developed a model using windows-based five-fold cross validation that combines both types of information (see Methods section). This hybrid model performs better than existing models and achieved maximum $85.92 \%$ sensitivity, $91.68 \%$ specificity, $90.14 \%$ accuracy, MCC 0.76 and AUC of 0.936 (Figure 4a). We also used sequence-based five-fold cross validation of tRNA-136 dataset, where we kept all the windows of any single tRNA into a same sub-set during five-fold cross validation and achieved almost equal performance $(83.13 \%$ Sensitivity, 92.36\% Specificity, 89.84\% accuracy and 0.75 $\mathrm{MCC}$ ) to the window-based five-fold cross validation.

To check whether over-representation of Pseudouridine (Y), Dihydrouridine (D) and 5-methyl-uridine (T) caused any SVM paramer over-fitting or not, we have used almost equal representation of different modifications. We randomly selected $30 \mathrm{Y}, 30 \mathrm{D}, 30 \mathrm{~T}$ and 92 all other uridine modifications from the tRNA-136 dataset for model development. On this randomized dataset, we have achieved $85.72 \%$ sensitivity, $97.34 \%$ specificity, 96.27\% accuracy, MCC 0.79 and AUC of 0.974 on the same previosuly optimized parameter (-z c -t 2 -g 0.05 -c 2 -j 2). It means there is no SVM parameter overfitiing during model development (Table 1).

\section{Classification of different uridine modifications}

In the tRNA-136 dataset, $\sim 72 \%$ of modified uridines belonged to either Pseudouridine or Dihydrouridine. 


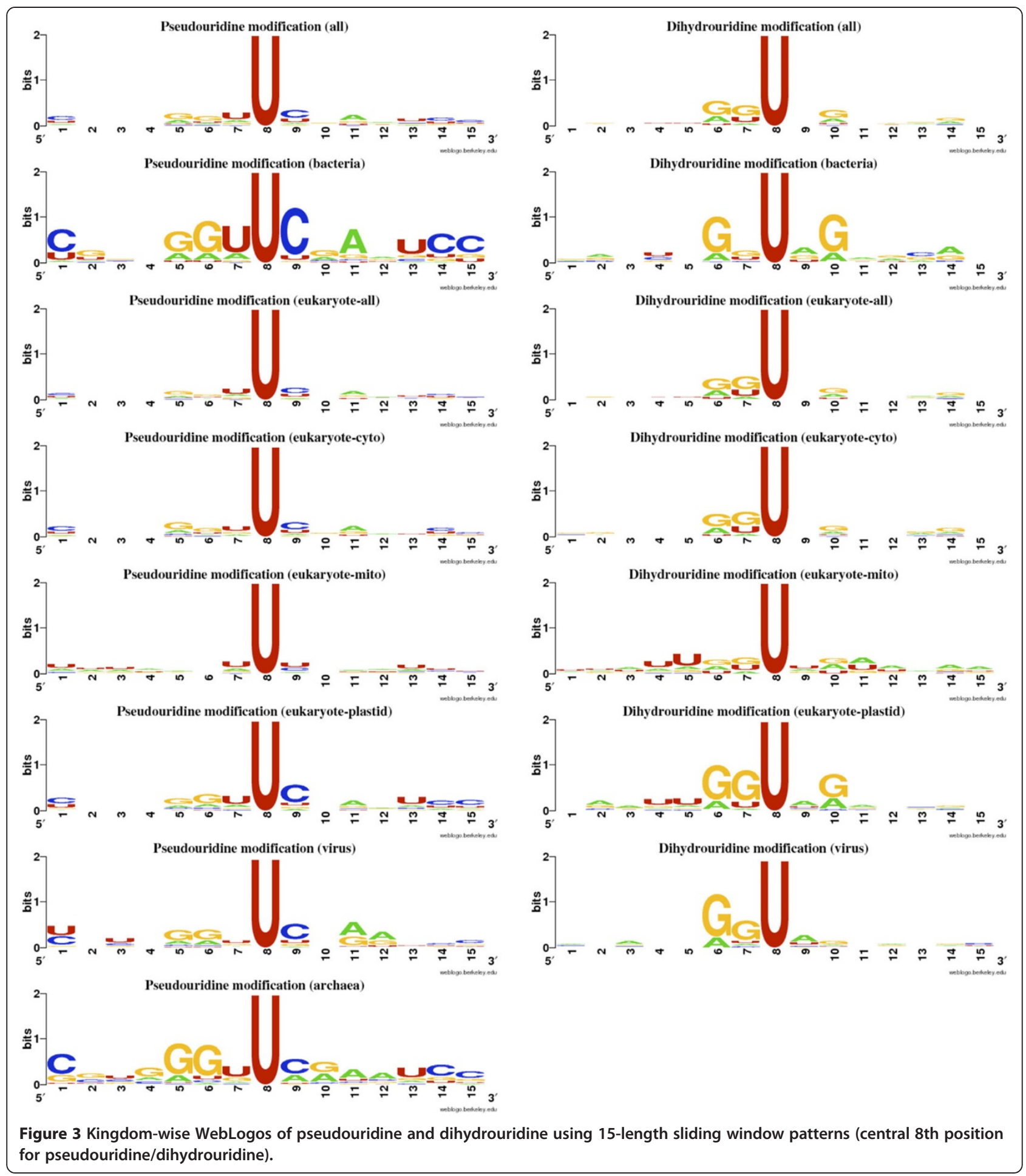

Therefore, we developed separate prediction models for these two major classes of UMs.

\section{Prediction of pseudouridine $(Y)$ modification}

We used patterns of Pseudouridine as positives and all other UMs as negatives. The maximum performance
MCC 0.93 and 0.96 was achieved using SVM models based on BPP and tRNAscan-SE based approach respectively. We achieved best threshold-independent performance AUC 0.986, 0.985 and 0.983 using SVM models based on BPP, tRNAscan-SE predicted structures and hybrid approach respectively (Figure 4b). 
(a) ROC Plot: Prediction of modified U

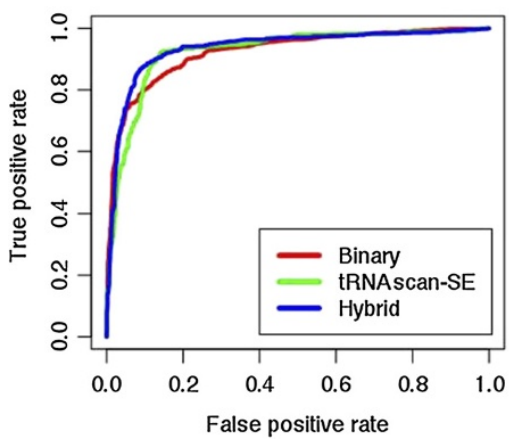

(b)

ROC Plot: Prediction of $Y$

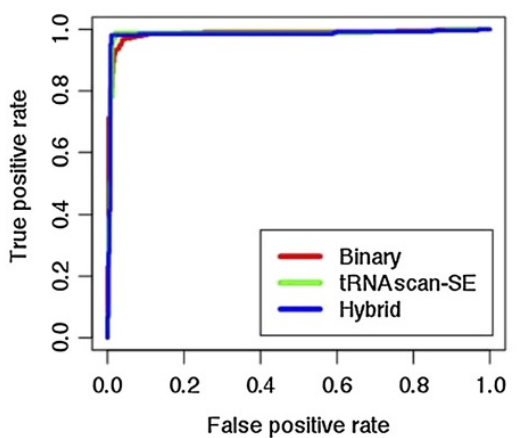

(c) ROC Plot: Prediction of D

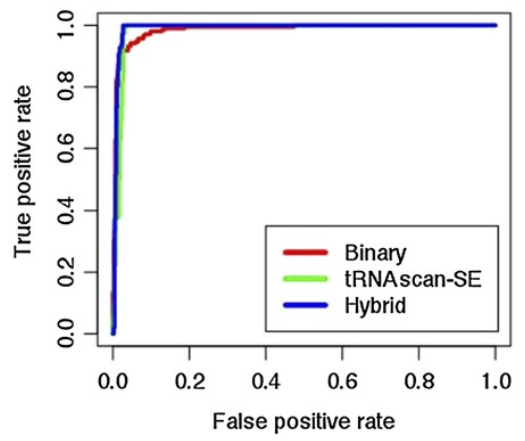

Figure 4 ROC plots showing prediction performances of (a) all uridine, (b) pseudouridine and (c) dihydrouridine modifications using Binary, tRNAscan-SE and Hybrid approaches on the tRNA-136 dataset.

Table 1 SVM performance on the complete tRNA-136 and randomly selected tRNA-136 dataset using the same parameter (-z c -t 2 -g 0.05 -c 2 -j 2)

\begin{tabular}{|c|c|c|c|c|c|c|c|c|}
\hline \multirow[t]{2}{*}{ Threshold } & \multicolumn{4}{|c|}{ Complete tRNA-136 dataset } & \multicolumn{4}{|c|}{ Randomized tRNA-136 dataset } \\
\hline & SN & SP & ACC & MCC & SN & SP & ACC & MCC \\
\hline-1.00 & 96.37 & 60.89 & 70.42 & 0.51 & 97.24 & 78.59 & 80.30 & 0.49 \\
\hline-0.90 & 95.31 & 70.16 & 76.91 & 0.58 & 96.68 & 85.80 & 86.80 & 0.58 \\
\hline-0.80 & 94.25 & 76.37 & 81.17 & 0.63 & 96.13 & 90.13 & 90.68 & 0.65 \\
\hline-0.70 & 93.64 & 80.42 & 83.97 & 0.67 & 93.36 & 92.96 & 93.00 & 0.70 \\
\hline-0.60 & 92.13 & 83.30 & 85.67 & 0.69 & 91.71 & 94.56 & 94.31 & 0.73 \\
\hline-0.50 & 91.37 & 85.14 & 86.81 & 0.71 & 89.52 & 95.73 & 95.16 & 0.76 \\
\hline-0.40 & 90.31 & 86.63 & 87.62 & 0.72 & 88.44 & 96.51 & 95.77 & 0.78 \\
\hline-0.30 & 89.40 & 87.80 & 88.23 & 0.73 & 87.34 & 96.89 & 96.02 & 0.78 \\
\hline-0.20 & 88.64 & 89.35 & 89.17 & 0.75 & 85.72 & 97.34 & 96.27 & 0.79 \\
\hline-0.10 & 87.43 & 90.57 & 89.73 & 0.75 & 84.07 & 97.67 & 96.42 & 0.79 \\
\hline 0.00 & 85.92 & 91.68 & 90.14 & 0.76 & 80.80 & 98.17 & 96.58 & 0.79 \\
\hline 0.10 & 83.80 & 92.57 & 90.22 & 0.75 & 75.82 & 98.56 & 96.47 & 0.78 \\
\hline 0.20 & 81.08 & 93.01 & 89.81 & 0.74 & 74.71 & 98.78 & 96.58 & 0.78 \\
\hline 0.30 & 79.72 & 93.62 & 89.90 & 0.74 & 71.44 & 98.89 & 96.37 & 0.77 \\
\hline 0.40 & 76.70 & 94.34 & 89.61 & 0.73 & 68.69 & 99.00 & 96.22 & 0.76 \\
\hline 0.50 & 73.67 & 95.28 & 89.49 & 0.72 & 62.69 & 99.11 & 95.77 & 0.72 \\
\hline 0.60 & 69.43 & 95.89 & 88.80 & 0.70 & 58.27 & 99.33 & 95.57 & 0.70 \\
\hline 0.70 & 65.20 & 96.67 & 88.23 & 0.69 & 53.87 & 99.33 & 95.16 & 0.67 \\
\hline 0.80 & 59.00 & 97.28 & 87.01 & 0.65 & 45.11 & 99.39 & 94.41 & 0.61 \\
\hline 0.90 & 51.59 & 97.78 & 85.39 & 0.60 & 32.40 & 99.45 & 93.30 & 0.50 \\
\hline 1.00 & 40.84 & 98.39 & 82.95 & 0.53 & 26.37 & 99.72 & 93.00 & 0.46 \\
\hline
\end{tabular}

Bold are performances with maximum MCC (Threshold 0.0 or close to 0.0 preferred).

Bold and italic are performances where gap between sensitivity and specificity are minimum. 


\section{Prediction of Dihydrouridine (D) modification}

The patterns of Dihydrouridines were used as positive and other patterns of UMs were used as negatives. The BPP approach achieved 0.90 MCC and 0.986 AUC whereas tRNAscan-SE based approach achieved 0.95 MCC and 0.985 AUC. The hybrid approach predicted all Dihydrouridines correctly (100\% sensitivity) with $97.36 \%$ specificity, 98.18\% accuracy, 0.96 MCC and 0.991 AUC values (Figure $4 \mathrm{c}$ ).

\section{Evaluation of developed models on the kingdom-wise independent datasets}

In the second step, we wanted to see the performance of a common prediction model (based on the tRNA-136 dataset) on the kingdom-wise independent datasets. We used newly added tRNA sequences (which were not used for the common prediction model development; see material and methods section) of MODOMICS databaset [40] update 2012, for the performance evaluation of previously developed model. We achieved 0.911, 0.949, 0.919, 0.910, 0.936, 0.789, 0.930 and 0.944 of AUC for the prediction of modified uridines of All, Archaea, Bacteria, Eukaryote-all, Eukaryote-cyto, Eukaryote-mito, Eukaryote-plastid and Viruses respectively (Figure 5a). The model was not good to predict UMs in the mitochondrial tRNAs because structure of 18 out of total 93 mitochondrial tRNAs were not predicted. Therefore, we also applied previously developed BPP approach based model (based on tRNA-136 dataset), where structural information was not required and achieved 0.892, 0.943, 0.916, 0.886, 0.919, 0.748, 0.892 and 0.945 of AUC for the prediction of modified uridines of All, Archaea, Bacteria, Eukaryote-all, Eukaryote-cyto, Eukaryote-mito, Eukaryote-plastid and Viruses respectively (Figure $5 \mathrm{~b}$ ). Here also prediction performance was low for mitochondrial tRNAs. In the most of cases performances were decreased when we applied BPP instead of hybrid approach. It means structural information provided important information for the both, common (all) and kingdom-wise predictions.

Hybrid approach based models achieved 0.84, 0.81, 0.90, $0.84,0.82,0.85,0.97$ and 0.75 of MCC for the pseudouridine prediction of All, Archaea, Bacteria, Eukaryote-all, Eukaryote-cyto, Eukaryote-mito, Eukaryote-plastid and Viruses respectively. In the dihydrouridine prediction, hybrid model achieved 0.90, 0.97, 0.89, 0.90, 0.88, 0.89 and 0.92 of MCC for the modified (dihydrouridine) uridines of All, Bacteria, Eukaryote-all, Eukaryote-cyto, Eukaryote-mito, Eukaryote-plastid and Viruses respectively.

\section{Kingdom-wise prediction model development}

In the third and last step, we used new kingdom-wise datasets from MODOMICS database [40] update 2012. It was shown in the previous approaches and evaluated by independent datasets that hybrid approach performed better if the structure predicted by tRNAscanSE otherwise BPP approach can also be use as an alternative. Therefore, we have developed kingdomspecific prediction models using tRNA-419 and tRNA471 datasets (See details in the material and method section). The tRNA-471 is a $50 \%$ non-redundant and containing 54 archaeal, 124 bacterial, 279 eukaryotic (142 cytosolic, 110 mitochondrial and 27 plastidic) and 14 viral tRNAs. First we developed a BPP approach based common prediction model for modified uridine prediction and achieved 0.917 of AUC. When we analyzed the kingdom-wise performance in this common prediction than we found 0.867, 0.901, 0.932, 0.946, $0.915,0.974$ and 0.837 of AUC for the Archaea, (a) Hybrid approach: prediction of modified $U$

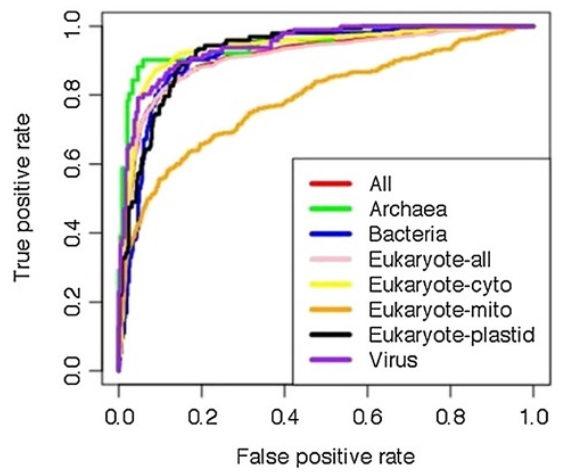

(b) Binary approach: prediction of modified $U$

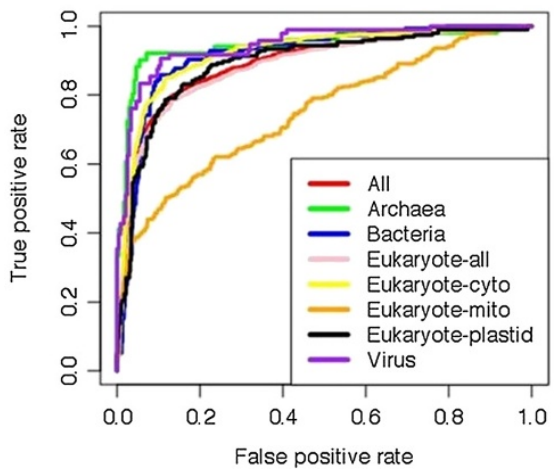

Figure 5 ROC plots showing kingdom-wise prediction performances of (a) hybrid and (b) binary approach on the independent datasets. 
Bacteria, Eukaryote-all, Eukaryote-cyto, Eukaryote-mito, Eukaryote-plastid and Viruses respectively (Figure 6a). In the kingdom-wise individual (separately for each kingdom/orgenelle) model development, we achieved 0.970, 0.907, 0.925, 0.949, 0.868, 0.883 and 0.867 of AUC for the Archaea, Bacteria, Eukaryote-all, Eukaryote-cyto, Eukaryote-mito, Eukaryote-plastid and Viruses respectively (Figure 6b). These kingdom-wise individual models were also developed for the pseudouridine and dihydrouridine. In the pseudouridine prediction, we achieved 0.974, 0.933, 0.987, 0.964, 0.963, 0.952, 0.975 and 0.880 of AUC for the All, Archaea, Bacteria, Eukaryote-all, Eukaryote-cyto, Eukaryote-mito, Eukaryote-plastid and Viruses respectively (Figure 6c). We also achieved 0.987, 0.997, 0.977, 0.981, 0.970, 0.977 and 0.895 of AUC for the dihydrouridine prediction of All, Bacteria, Eukaryote-all, Eukaryote-cyto, Eukaryote-mito, Eukaryote-plastid and Viruses respectively (Figure 6d).
In the hybrid approach, we used tRNA-419 (50\% non-redundant) dataset instead of tRNA-471, because tRNAscan-SE software was not predicted structures of 52 tRNA sequences. The tRNA-419 dataset containing 53 archaeal, 121 bacterial, 233 eukaryotic (114 cytosolic, 92 mitochondrial and 27 plastidic) and 12 viral tRNAs. A common prediction model for modified uridine prediction achieved 0.941 of AUC in comparison to 0.917 AUC of BPP approach. In this performance of 0.941 AUC, kingdom-wise prediction performances were 0.962, $0.930,0.962,0.962,0.962,1.00$ and 0.952 of AUC for the Archaea, Bacteria, Eukaryote-all, Eukaryote-cyto, Eukaryote-mito, Eukaryote-plastid and Viruses respectively (Figure 7a). In the kingdom-wise individual model development, we achieved 0.987, 0.931, 0.953, 0.959, $0.940,0.924$ and 0.915 of AUC for the Archaea, Bacteria, Eukaryote-all, Eukaryote-cyto, Eukaryote-mito, Eukaryote-plastid and Viruses respectively (Figure 7b). (a)

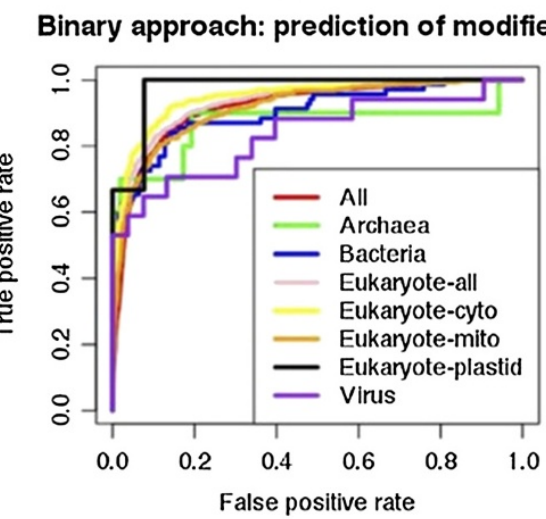

(b)

Binary approach: prediction of modified $U$

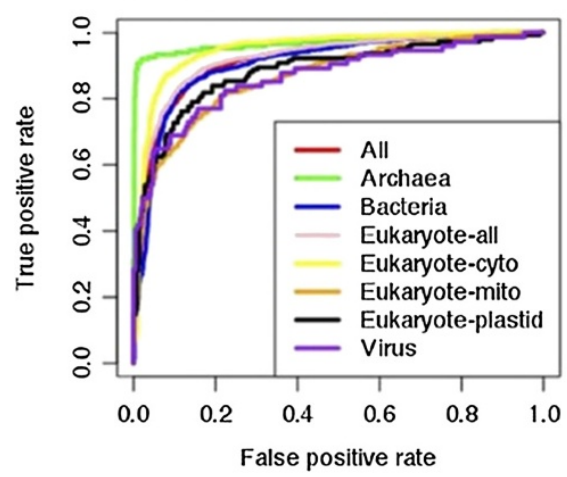

(c)

\section{Binary approach: prediction of $Y$}

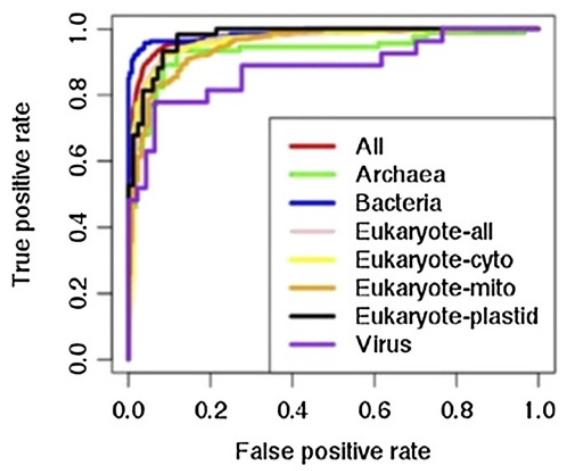

(d)

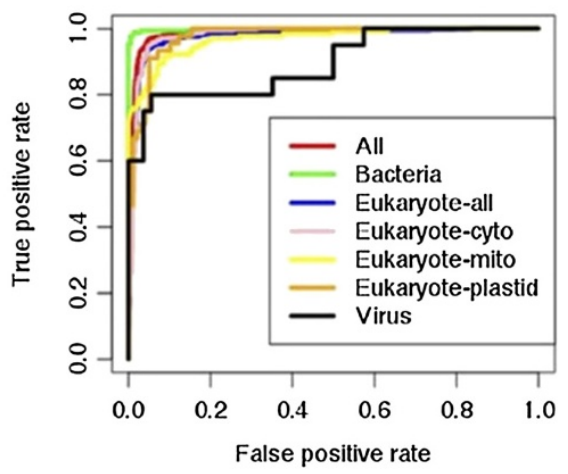

Figure 6 ROC plots showing BPP approach based prediction performances on the kingdom-wise datasets (tRNA-471) for the prediction of (a) uridine modifications on the common prediction model, (b) uridine modifications on the kingdom-wise individual (separately for each kingdom/orgenelle) models, (c) Pseudouridine modification on the kingdom-wise separate models and (d) Dihydrouridine modification on the kingdom-wise separate models. 
(a)

Hybrid approach: prediction of modified $U$

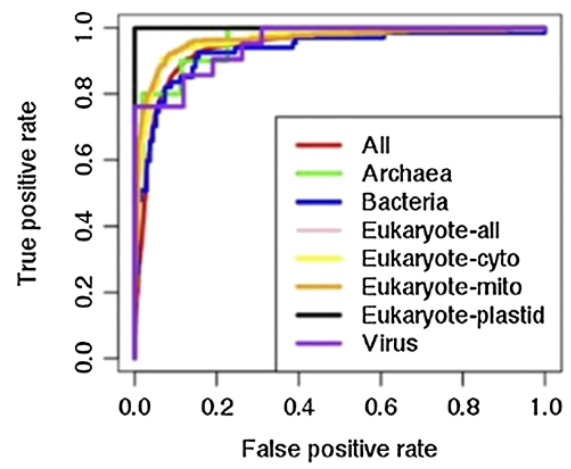

(b)

Hybrid approach: prediction of modified $U$

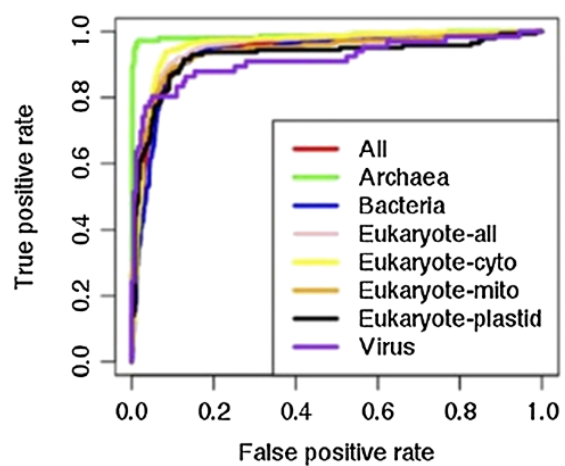

(c)

Hybrid approach: prediction of $Y$

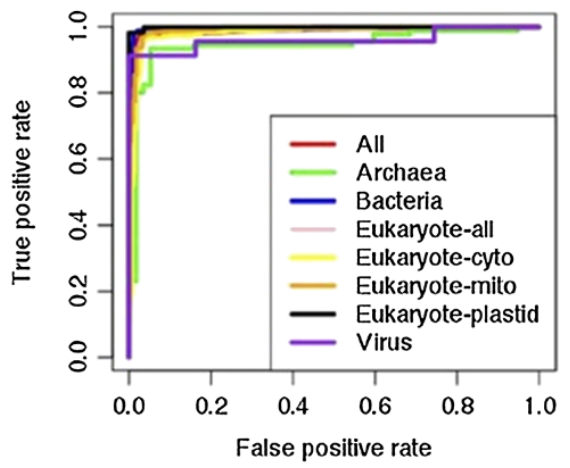

(d)

Hybrid approach: prediction of D

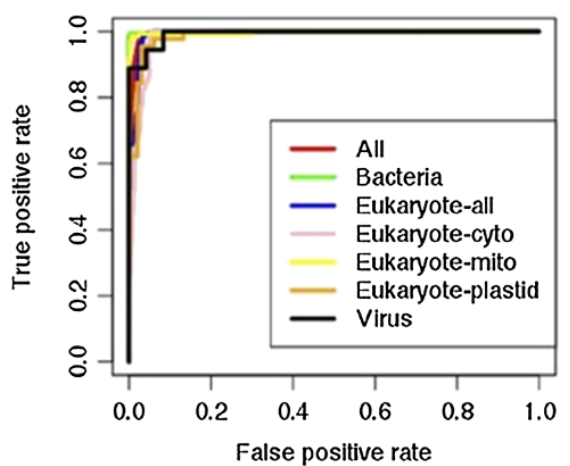

Figure 7 ROC plots showing hybrid approach based prediction performances on the kingdom-wise datasets (tRNA-419) for the prediction of (a) uridine modifications on the common prediction model, (b) uridine modifications on the kingdom-wise individual (separately for each kingdom/orgenelle) models, (c) Pseudouridine modification on the kingdom-wise separate models and (d) Dihydrouridine modification on the kingdom-wise separate models.

These kingdom-wise individual models were also developed for the pseudouridine and dihydrouridine. The pseudouridine prediction performances were $0.986,0.945,0.996,0.985$, 0.981, 0.992, 0.999 and 0.961 of AUC for the All, Archaea, Bacteria, Eukaryote-all, Eukaryote-cyto, Eukaryote-mito, Eukaryote-plastid and Viruses respectively (Figure 7c). This hybrid approach achieved 0.994, 0.999, 0.990, 0.983, 0.997, 0.988 and 0.993 of AUC for the dihydrouridine prediction of All, Bacteria, Eukaryote-all, Eukaryote-cyto, Eukaryote-mito, Eukaryote-plastid and Viruses respectively (Figure $7 \mathrm{~d}$ ). The dihydrouridine modifications were absent in the archaeal tRNA sequences. These results showed that Hybrid approach performed better in comparison to BPP approach, whether kingdom-wise performances in the common method or individually developed methods. Hybrid approach also performed better than BPP for the prediction of pseudouridine and dihydrouridine.

Although, our main focused was to predict UMs in tRNA sequences and further classify them into pseudouridine and dihydrouridine modifications but we also tried to develop method for the third ( $11 \%$ of all UMs) most abundant UM- 5-methyl-uridine.

\section{Prediction of 5-methyl-uridine ( $\mathrm{T}$ ) modification}

The tRNA-136 dataset contains 14.2\% UMs as 5-methyluridines. We analyzed kingdom-wise patterns and found that this modification is present only at a well-known conserved site GTYC (GUUC in the WebLogo) of the T-Stem Loop (Additional file 1: Figure S10). Therefore, it is easy to predict this modification, if boundaries of loops (mainly VL and TSL) are correctly predicted in the tRNA. The patterns of 5-methyl-uridine were used as positives and other UMs were used as negatives for developing SVMbased models. First we have used tRNA-136 and developed common model for the prediction of 5-methyl-uridines. In the threshold-dependent performances, BPP approach achieved $97.9 \%$ sensitivity, $97.36 \%$ specificity, $97.43 \%$ accuracy and $0.90 \mathrm{MCC}$ whereas tRNAscan-SE achieved 96.84\% sensitivity, $94.54 \%$ specificity, $94.86 \%$ accuracy and 
0.82 MCC. Hybrid approach increased the performance and achieved $94.74 \%$ sensitivity, $98.42 \%$ specificity, $97.88 \%$ accuracy and $0.92 \mathrm{MCC}$. The threshold-independent performance of BPP, tRNAscan-SE and Hybrid approaches achieved 0.991, 0.971 and 0.993 AUC values (Additional file 1: Figure S11a). On the independent datasets (which were not used in the tRNA-136 dataset), Hybrid approach based model achieved 0.90, 0.97, 0.93, 0.93, 0.98, 0.90 and 0.78 of MCC for the 5-methyl-uridine prediction of All, Bacteria, Eukaryote-all, Eukaryote-cyto, Eukaryote-mito, Eukaryote-plastid and Viruses respectively. Finally, we applied BPP (tRNA-471 dataset) and hybrid (tRNA-419 dataset) approaches and achieved 0.993, 0.997, 0.996, 0.997, 0.997, 1.00 and 0.985 of AUC for BPP (Additional file 1: Figure 11b) and 0.993, 0.995, 0.996, 0.996, 0.998, 1.00 and 0.985 of AUC for hybrid approach (Additional file 1: Figure 11c) for the All, Bacteria, Eukaryote-all, Eukaryote-cyto, Eukaryote-mito, Eukaryoteplastid and Viruses respectively. The 5-methyl-uridine modification was absent in the archaeal tRNA sequences.

\section{Prediction of other uridine modifications}

To see whether SVM based machine learning can discriminate these three (Y,D and T) modifications from other UMs (remaining $12 \%$ ) or not, we applied same above-mentioned strategy. We used tRNA-136 dataset and patterns of $\mathrm{Y}, \mathrm{D}$ and $\mathrm{T}$ as negatives and patterns of other UMs as positives. The BPP approach performed $66.26 \%$ sensitivity, $98.42 \%$ specificity, $93.95 \%$ accuracy and 0.73 MCC. The structural information of tRNAscan-SE based approach achieved $52.16 \%$ sensitivity, 99.82\% specificity, 93.19\% accuracy and 0.69 MCC. The Hybrid approach increased performance significantly and achieved $75.96 \%$ sensitivity, $97.89 \%$ specificity, $94.86 \%$ accuracy and 0.78 MCC. The threshold-independent performance of BPP, tRNAscan-SE and Hybrid approaches achieved 0.924, 0.868 and 0.922 AUC values (Additional file 1: Figure S12a).

On the independent datasets, Hybrid approach based model achieved 0.58, 0.77, 0.86, 0.41, 0.38, 0.49, 0.53 and 0.64 of MCC for the other modified (except Y, D and T) uridines of All, Archaea, Bacteria, Eukaryote-all, Eukaryotecyto, Eukaryote-mito, Eukaryote-plastid and Viruses respectively. The BPP (tRNA-471 dataset) and hybrid (tRNA-419 dataset) approaches were achieved 0.905, $0.933,0.974,0.845,0.847,0.916,0.905$ and 0.860 of AUC for BPP (Additional file 1: Figure 12b) and 0.925, $0.947,0.976,0.889,0.877,0.967,0.867$ and 0.995 of AUC for hybrid approach (Additional file 1: Figure 12c) for the All, Archaea, Bacteria, Eukaryote-all, Eukaryotecyto, Eukaryote-mito, Eukaryote-plastid and Viruses respectively. The prediction performances were low in comparison to Y, D and T because here we used total 19 different types of modifications together as positives. In order to implement the prediction model in the form of web-server, it was necessary to develop a separate prediction model for other UMs (remaining 12\%).

In conclusion, SVM-based prediction modules performed better with hybrid approach of BPP and tRNAscan-SE based structural information. The details of all results given in an excel file (see Additional file 2), contain results of all approaches, window sizes at all (-1.0 to 1.0) thresholds and ROC graphs.

\section{Discussion}

In present study, we retrieved information of 218 and 642 modified tRNAs from MODOMICS database [39,40]. Initially, we analyzed all 642 tRNA modifications and observed that majority $(55.85 \%)$ of modifications were uridinederived (Figure 1) and $29.27 \%$ uridines of all uridines were modified. Therefore, we selected only UMs for further study. The kingdom-wise differences between flanking nucleotides of modified and unmodified uridines were observed (Figure 2). It may be due to the pattern-wise preference of modifying enzymes. In past, sliding windowbased approach was widely used for nucleotide/residue level predictions [46]. It requires complete optimization of all window sizes for every prediction. We created different lengths (3-25) of sliding window patterns and various approaches of compositions; BPP and structural information were applied. First we used tRNA-136 dataset for the common prediction model and evaluated using 5-fold cross validation technique. In compositions based input features of MNC, DNC and TNC achieved AUC of 0.76, 0.840 and 0.865 respectively. The $\mathrm{BPP}$ increased the prediction performance to AUC of 0.924 AUC because it provided information of nucleotides with their positions whereas compositions based approaches have only frequencies or one/two neighboring nucleotide information. All tRNA fold into well defined structures with some regions and loops more prone to modification thereby making the structural information useful for prediction. Consequently, secondary structures provided by tRNAscan-SE software achieved AUC of 0.925. When the structural information of tRNAscan-SE software predicted boundaries of different loops was combined with positional information of nucleotides in the form of BPP; performance increased significantly and achieved AUC of 0.936 (Figure 4a). We have also analyed the effect of window size on the prediciton performance and found that performance continously increased in 3-15 window size and saturated between the 17-25 window sizes (Figure 8).

In the second step, we evaluated previously developed (based on tRNA-136) models on the independent datasets and analyzed the kingdom wise performances. Hybrid approach performed well for All, Archaea, Bacteria, Eukaryoteall, Eukaryote-cyto, Eukaryote-plastid and Viruses but not performed for Eukaryote-mito (Figure 5). It may be because tRNA-136 contained only 18 mitochondrial tRNAs of 


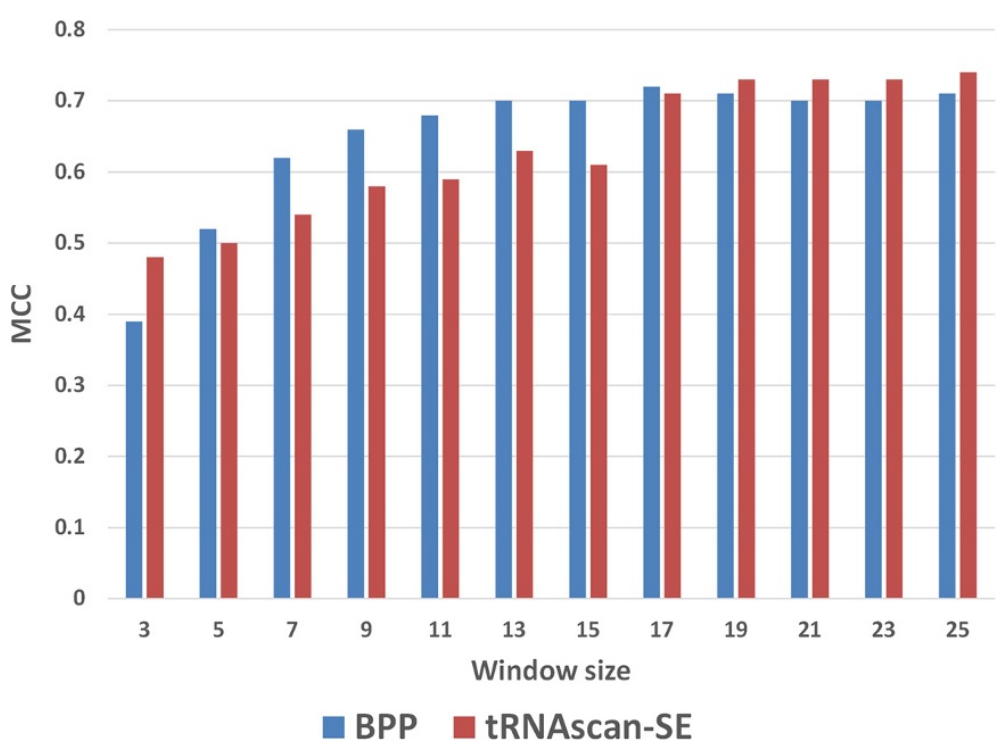

Figure 8 Prediction performance (MCC) of BPP and tRNAscan-SE based approaches using different window sizes.

Saccharomyces cerevisiae and developed model was not good enough to predict UMs in the mitochondrial tRNAs from other eukaryotes. Although, mitochondrial tRNAs have evolutionary connection with bacterial tRNAs but our results showed that prokaryotic datasets based model cannot predict the uridine modifications in the mitochondrial tRNAs.

In the last step, we used BPP (based on tRNA-471 dataset) and hybrid (based on tRNA-419 dataset) approach for the final kingdom-specific prediction models and evaluated using five-fold cross validation technique. The hybrid approach performed better than simple BPP approach in all kingdoms. It means structural information provided important information, when it integrated with BPP (Figures 6 and 7). Hybrid approach achieved 0.941 AUC for all tRNAs but when applied separately for Archaea and Eukaryotes, it increased to the AUC of 0.987 and 0.953 respectively. We found that hybrid approach of BPP and tRNAscan-SE was efficient not only to predict UMs but also to classify them into pseudouridine and dihydrouridine (Figure 7). The BPP approach can also be useful as an alternative, if structure of tRNA is not available (Figure 6). We also developed models for the third most abundant ( $11 \%$ of all UMs) 5 -methyl-uridines UMs.

A recent study of Novoa et al. [38] showed that additional information of two modifications $\left(\mathrm{I}_{34}\right.$ and $\left.\mathrm{xo}^{5} \mathrm{U}_{34}\right)$ improved correlation between codon usage and tRNA gene frequencies in all kingdoms significantly. Modifications in ASLs are very important given the fact that modified $U_{34}$ acts as proton donor/acceptor, coordinates metal ions and has great diverse chemistry [27] ultimately affecting codonanticodon recognition. The ASLs, DSLs, TSLs and VLs of different tRNAs vary in sequence and the type of modification. Modification of seven-nucleotide ASLs (especially wobble 34 and purine 37 site) is more important because global conformation of ASLs decides entry of anticodon domain into the ribosomal A-site [13] and affects translation speed [47]. Data availability is the main criterion for the development of any prediction model and the number of uridine modifications $(55.85 \%)$ is very high in comparison to guanine (19.71\%), adenine $(12.28 \%)$ and cytosine (10.41\%) modifications. Therefore, we developed prediction models for UMs only. Many RNA modifications are not essential for cell survival. Probably these modifications are less important or not fully explored yet considering the fact that many DNA and protein modifications are also not essential. Precise roles of most of tRNA modifications are poorly understood and their industrial applications are still unexplored. Pseudouridine provides rigidity whereas dihydrouridine is the only non-aromatic nucleoside and provides flexibility to the tRNA structure. In this era of synthetic biology, better understanding of tRNA modifications will help in the better tRNA designing, incorporation of novel amino acids and production of new proteins. In particular, biochemists have great opportunity to play with the chemistry of wobble base and expand amino acid boundaries.

\section{Conclusion}

To conclude, the present study is a systematic attempt to predict and classify UMs in tRNA sequences. We developed separate and kingdom-wise predictors for the prediction of UMs and thereafter classify them into Pseudouridines, Dihydrouridines and 5-methyl-uridine and other UMs. We found that hybrid approach of binary and structural information is most suitable for the 
SVM based prediction of UMs. These prediction modules have been implemented in a 'tRNAmod' web-server. This server can predict and classify UMs from tRNA sequences or whole genome of any organism.

\section{Methods \\ Datasets}

In this study, two different version of MODOMICS database update 2008 [39] and update 2012 [40] have been used. Update 2008 and 2012 of MODOMICS database were containing 218 and 642 tRNA (modified) sequences respectively.

\section{MODOMICS database update 2008}

We extracted 43 different types of modification in total 218 (35 Bacillus subtilis, 47 Escherichia coli, 41 Halobacterium volcanii, 29 Mycoplasma capricolum and 66 Saccharomyces cerevisiae) modified tRNA sequences from MODOMICS database [39]. Out of the total 17088 bases in these sequences, 1674 bases are modified and 15414 are unmodified. The base-specific contribution in modifications of uridine, guanine, adenine and cytosine are $61.4 \%, 17.2 \%, 11.2 \%$ and $10.2 \%$ respectively.

\section{tRNA-136}

To develop a prediction method, it is important to create non-redundant dataset because prediction of modified base in diverse sequences is a major challenge. In the case of RNA sequences $50 \%$ non-redundancy level is sufficient to evaluate prediction model and also most of tools are not reliable for generating more stringent redundancy level for nucleotide sequences. Therefore, we created 50\% non-redundant (NR) dataset of 136 (16 Bacillus subtilis, 33 Escherichia coli, 31 Halobacterium volcanii, 9 Mycoplasma capricolum and 47 Saccharomyces cerevisiae) tRNA sequences from MODOMICS database update 2008 using BLASTCLUST software and termed it as 'tRNA-136'. The tRNA-136 dataset contains a total of 10654 bases, out of which 1095 are modified (10.28\%) and 9559 are unmodified bases. It includes 40 different types of modifications. In tRNA-136 dataset, 661 (60.4\%) modified-bases are uridine-derived. Most of uridine modifications belong to Pseudouridine (40.5\%), Dihydrouridine (31.3\%), 5-methyl-uridine (14.2\%) and remaining $14 \%$ are other UMs.

\section{MODOMICS database update 2012}

We extracted total 642 (413 eukaryotic, 152 bacterial, 60 archaeal and 17 viral) modified tRNA sequences from MODOMICS database [40], which contained 60 type of modifications from 77 different organisms (49 eukaryotes, 17 bacteria, 7 archaea and 4 viruses). Total $\sim 13 \%$ bases were modified and uridine-, guanine-, adenineand cytosine-derived modified bases were 55.85\%, 19.71\%,
$12.28 \%$ and $10.41 \%$ of total modified bases respectively (Additional file 1: Table S1).

\section{Independent datasets}

In order to create kingdom-wise independent datasets, we used only newly updated (organism-wise) tRNA sequences of MODOMICS database update 2012 and excluded all the tRNA sequences (all the tRNAs of Bacillus subtilis, Escherichia coli, Haloferax volcanii (formerly named as Halobacterium volcanii), Mycoplasma capricolum and Saccharomyces cerevisiae) of MODOMICS update 2008. In this way, we created kingdom-wise independent datasets of all 407 tRNAs, which contains 19 archaeal, 41 bacterial, 330 eukaryotic (199 cytosolic, 93 mitochondrial and 38 plastidic) and 17 viral tRNA sequences. These independent datasets were used for the kingdom-wise performance evaluation of common prediction model (based on tRNA-136).

\section{tRNA-471}

We used all 642 tRNAs of MODOMICS database update 2012 and created 50\% non-redundant (NR) dataset of 471 tRNA sequences using BLASTCLUST software and termed it as 'tRNA-471'. It contains 54 archaeal, 124 bacterial, 279 eukaryotic (142 cytosolic, 110 mitochondrial and 27 plastidic) and 14 viral tRNA sequences. We used these kingdom-wise datasets for the model development of BPP approach.

\section{tRNA-419}

In the hybrid approach, tRNAscan-SE software was not predicted structure of 52 tRNAs from tRNA-471 dataset. Therefore, we created separate dataset of 419 tRNAs and termed as 'tRNA-419'. It contains 53 archaeal, 121 bacterial, 233 eukaryotic (114 cytosolic, 92 mitochondrial and 27 plastidic) and 12 viral tRNA sequences. In the hybrid approach, we used these kingdom-wise datasets for the prediction model development.

Both the dataset, data-218 (MODOMICS database update 2008) and data-642 (MODOMICS database update 2012) have been provided in the Additional file 3 and Additional file 4 respectively. The nomenclature of modification used from MODOMICS database, which has been also provided in the Additional file 1: Table S1.

\section{Creation of sliding windows}

In past, sliding window-based strategies have been successfully applied for residue level predictions [46]. Thus, we created and optimized different lengths of window size (325) of odd numbers (eg. 3, 5, 7,.., 25). If the central nucleotide of window pattern is modified then it was assigned as positive pattern otherwise assigned as negatives. In this way, we created window patterns for each nucleotide in the tRNA-136 dataset. To generate fixed length window 
size of terminal nucleotides, we added a dummy ' $\mathrm{X}$ ' nucleotide in both terminals of each sequence. The number of dummy nucleotides was calculated with (L-1)/2 formula (where $\mathrm{L}$ is the length of pattern). In this study, we used maximum window size 25 because the average length of tRNA is 77 (each containing 3 loops) and large window sizes are not advisable for better machine learning. Thus, we preferred well performing small window to large window sizes.

\section{Compositions-based approaches}

In this approach, we calculated mono-nucleotide composition (MNC), di-nucleotide composition (DNC) and tri-nucleotide composition (TNC) of tRNA sequences. After adding dummy ' $\mathrm{X}$ ' at both terminals, total number of nucleotides reached to five (A, C, G, U and X). The $\mathrm{MNC}, \mathrm{DNC}$ and TNC generated input features of 5 (A, C, G, U and X), 25 (AA, AC, AG, CG, AU,..., XX) and 125 (AAA, AAC, AAG,..., XXX) dimensions of vector.

\section{Binary approach}

As discussed above, positive and negative patterns of sliding windows were created but numerical representation of these patterns is necessary for SVM-based machine learning. Thus, we applied binary profile of patterns (BPP) approach, which gives nucleotide (or amino acids) information as well their positional (sequential) information [46]. In BPP, we represented A, C, G, U and X nucleotides with $\{1,0,0,0,0\},\{0,1,0,0,0\},\{0,0,1,0,0\},\{0,0,0,1,0\}$ and $\{0,0,0,0,1\}$ respectively. The number of total input features generated by BPP was five times more than used window size (e.g. 17-length window generates total $85(17 \times 5)$ input features).

\section{Structure-based approaches}

All tRNA sequences fold into well-defined structures, thus structural information was used for SVM-based machine learning. The secondary structures of all tRNAs were predicted using three different software: RNAfold [43], IPknot [44] and tRNAscan-SE [45]. We converted all the secondary structural information into binary pattern for prediction model development. Here also dummy ' $\mathrm{X}$ ' were used for the terminal nucleotides in order to create fixed length patterns. RNAfold gives three types of secondary structures, thus we represented small open bracket, small close bracket, dot and $X$ as $\{1,0,0,0\},\{0,1,0,0\},\{0,0,1,0\}$ and $\{0,0,0,1\}$ respectively. IPknot predicted five different types secondary structures, which include additional information of pseudoknots in the form of square brackets (open and close). Thus, binary representations of small open bracket, small close bracket, square open bracket, square close bracket, dot and dummy $X$ were $\{1,0,0,0,0\}$, $\{0,1,0,0,0\},\{0,0,1,0,0\},\{0,0,0,1,0\}$ and $\{0,0,0,0,1\}$ respectively. The tRNAscan-SE predicts tRNA secondary structures specifically and gives three different types secondary structures. We represented $<,>$, dot and $\mathrm{X}$ in the binary form of $\{1,0,0,0\},\{0,1,0,0\},\{0,0,1,0\}$ and $\{0,0,0,1\}$ respectively. In this way, we converted all the secondary structural information into machine learning input format and developed separate prediction model for each software.

\section{Hybrid approach}

The hybrid approach is an integration of two or more approaches. In this study, we observed that binary information of BPP (85 features of 17-length window) and predicted structures of tRNAscan-SE (76 input features of 19-length window) performed well individually. Thus, we integrated these two approaches and created $161(85+$ 76) input features. This hybrid approach, provided both nucleotide-wise positional information and structural information further improving prediction performance.

\section{Support vector machine}

SVM is widely used and highly successful machine learning technique for the biological predictions [48,49]. It is based on the structural risk minimization principle of statistical learning theory. SVMs are a set of supervised learning methods, which can be used for both classification and regression mode [50]. We implemented SVM ${ }^{\text {light }}$ (version 6.02) package for the development of all prediction models [51]. It provides several parameters and kernels (e.g. linear, polynomial, radial basis function, and sigmoid) or any user-defined kernel. The svm_learn and svm_classify are two main softwares in this package. First, we used svm_learn for training of known examples and building of prediction models. After training, learned models predicted unknown examples (in five-fold crossvalidation) using svm_classify. We tried various parameters and kernels and found that radial basis function (RBF) kernel performed well in all cases.

\section{Five-fold cross validation}

In past, various prediction performance evaluation techniques have been applied such as leave-one out crossvalidation (LOOCV) or jack-knife test, n-fold cross validation technique [52]. Though, jack-knife test is best for performance evaluation but it is a time-consuming process. Thus, we used 5-fold cross validation technique, which is highly used in the performance evaluation of biological predictions $[53,54]$. In 5-fold cross validation, we divided both positive and negative samples into five subsets separately. We created five sets and each set containing one positive and one negative subset. The four subsets have been used for training and the remaining fifth subset was used for testing and calculating the performance. This step was repeated five times in such a way that each subset 
was used once for testing. The final performance is an average performance of all five testing sets.

\section{Evaluation parameters}

The prediction performance of each model was calculated in the form of sensitivity (Equation 1), specificity (Equation 2), accuracy (Equation 3) and MCC (Equation 4) values. These are well-established evaluation parameters for biological prediction [54].

$$
\begin{aligned}
& \text { Sensitivity }=\frac{T P}{T P+F N} \times 100 \\
& \text { Specificity }=\frac{T N}{T N+F P} \times 100 \\
& \text { Accuracy }=\frac{T P+T N}{T P+F P+T N+F N} \times 100 \\
& M C C=\frac{(T P \times T N)-(F P \times F N)}{\sqrt{[T P+F P][T P+F N][T N+F P][T N+F N]}}
\end{aligned}
$$

Where TP, TN, FP and FN are True Positives, True Negative, False Positives and False Negatives respectively.

Above-mentioned evaluation parameters are thresholddependent so we also calculated threshold-independent performance in terms of Area Under Curve (AUC) values using Receiver Operating Curve (ROC) plots. It is a plot between true positive rate and false positive rate.

The tRNAmod web-server gives probability score for each prediction. To calculate this score we used Equation 5, where SVM score of more than 1.5 and less than -1.5 was fixed to 1.5 and -1.5 respectively.

$$
\text { Probability score }=\frac{\text { SVM score }+1.5}{3} \times 9
$$

The probability score range varies from $0-9$ only. We adopted this strategy because it is easy to display this probability score with tRNA sequence in tRNAmod web-server.

\section{Description of tRNAmod web-server}

A user-friendly web-server tRNAmod was developed for the kingdom-wise prediction of UMs in tRNA sequence. There are two different prediction options available in tRNAmod, (1) Sequence-level prediction and (2) Genomewide prediction. In the sequence-level prediction, it requires tRNA sequences in FASTA format and it will directly predict UMs in the given tRNAs. In the Genome-wide prediction, it requires whole genome sequence (also in FASTA fromat). First, it will extract tRNA sequences from the submitted genome using tRNAscan-SE [45] software then tRNAmod will predict UMs in extracted tRNA sequences. It will show the probability score (ranges 0-9) for each predicted UMs. The server shows secondary structure information of tRNA using tRNAscan-SE. A java-based applet VARNA [55] also has been implemented for structure visualization of tRNA, where predicted UMs will be highlighted in the tRNA structure. The tRNAmod is freely available for the help of global scientific community and is available at http://crdd.osdd.net/raghava/trnamod.

\section{Additional files}

Additional file 1: Figure S1. Weblogo of tRNA sequences in the standard representation of 1-99 positions. Figure S2. Kingdom-wise percent distribution of different base-specific modifications. Figure S3. A ROC plot showing prediction performances of uridine modifications of different window sizes using MNC approach. Figure S4. A ROC plot showing prediction performances of uridine modifications of different window sizes using DNC approach. Figure S5. A ROC plot showing prediction performances of uridine modifications of different window sizes using TNC approach. Figure S6. A ROC plot showing prediction performances of uridine modifications of different window sizes using binary approach. Figure S7. A ROC plot showing prediction performances of uridine modifications of different window sizes using RNAfold based approach. Figure S8. A ROC plot showing prediction performances of uridine modifications of different window sizes using IPknot based approach. Figure S9. A ROC plot showing prediction performances of uridine modifications of different window sizes using tRNAscan-SE based approach. Figure S10.

Kingdom-wise WebLogos of 5-methyl-uridine using 15-length sliding window patterns (central 8th position for 5-methyl-uridine). Figure S11. ROC plots showing performances for the prediction of 5-methyluridine (T) on the (a) tRNA-136 dataset, (b) BPP appraoch of tRNA-471 dataset and (c) hybrid approach of tRNA-419 dataset. Figure S12. ROC plots showing performances for the prediction of other uridines (except Y, D and T) modifications on the (a) tRNA-136 dataset, (b) BPP appraoch of tRNA-471 dataset and (c) hybrid approach of tRNA-419 dataset. Table S1. Modification-wise distribution of 642 tRNAs of the MODOMICS database. The nomenclature of modification used from MODOMICS database.

Additional file 2: Contains the detailed results of different approaches at different window sizes and thresholds.

Additional file 3: Contains the information of 218 modified tRNA from MODOMICS database update 2008.

Additional file 4: Contains the information of 642 modified tRNA from MODOMICS database update 2012.

\section{Competing interests}

The authors declare that they have no competing interests.

\section{Authors' contributions}

BP created dataset, developed the SVM models, created the backend web server and the front end user interface. GPSR conceived the project, coordinated it and refined the manuscript drafted by BP. All the authors have read and approved final manuscript.

\section{Acknowledgments}

Authors are thankful to funding agencies Council of Scientific and Industrial Research (project OSDD and GENESIS BSC0121) and Department of Biotechnology (project BTISNET), Government of India. This report has Institute of Microbial Technology (IMTECH) communication no. 055/2012.

\section{Author details}

${ }^{1}$ Bioinformatics Centre, CSIR-Institute of Microbial Technology, Sector 39A, Chandigarh, India. ${ }^{2}$ Present address: Department of Computational Medicine and Bioinformatics, University of Michigan, Ann Arbor, MI 48109, USA.

Received: 24 April 2014 Accepted: 24 September 2014

Published: 2 October 2014 


\section{References}

1. Helm M: Post-transcriptional nucleotide modification and alternative folding of RNA. Nucleic Acids Res 2006, 34(2):721-733.

2. Helm M, Brulé H, Degoul F, Cepanec C, Leroux JP, Giegé R, Florentz C: The presence of modified nucleotides is required for cloverleaf folding of a human mitochondrial tRNA. Nucleic Acids Res 1998, 26:1636-1643.

3. Motorin Y, Helm M: tRNA stabilization by modified nucleotides. Biochemistry 2010, 49:4934-4944.

4. Chernyakov I, Whipple JM, Kotelawala L, Grayhack EJ, Phizicky EM: Degradation of several hypomodified mature tRNA species in Saccharomyces cerevisiae is mediated by Met22 and the 5'-3' exonucleases Rat1 and Xrn1. Genes Dev 2008, 22:1369-1380.

5. Alexandrov A, Chernyakov I, Gu W, Hiley SL, Hughes TR, Grayhack EJ, Phizicky EM: Rapid tRNA decay can result from lack of nonessential modifications. Mol Cell 2006, 21:87-96.

6. Kadaba S, Krueger A, Trice T, Krecic AM, Hinnebusch AG, Anderson J: Nuclear surveillance and degradation of hypomodified initiator tRNAMet in S. cerevisiae. Genes Dev 2004, 18:1227-1240.

7. Agris PF: Decoding the genome: a modified view. Nucleic Acids Res 2004, 32:223-238

8. Agris PF, Vendeix FAP, Graham WD: tRNA's wobble decoding of the genome: 40 years of modification. J Mol Biol 2007, 366:1-13.

9. Dalluge JJ, Hashizume T, Sopchik AE, McCloskey JA, Davis DR: Conformational flexibility in RNA: the role of dihydrouridine. Nucleic Acids Res 1996, 24:1073-1079.

10. Gustilo EM, Vendeix FA, Agris PF: tRNA's modifications bring order to gene expression. Curr Opin Microbiol 2008, 11:134-140.

11. Krüger MK, Pedersen S, Hagervall TG, Sørensen MA: The modification of the wobble base of tRNAGlu modulates the translation rate of glutamic acid codons in vivo. J Mol Biol 1998, 284:621-631.

12. Yarian C, Townsend H, Czestkowski W, Sochacka E, Malkiewicz AJ, Guenther R, Miskiewicz A, Agris PF: Accurate translation of the genetic code depends on tRNA modified nucleosides. J Biol Chem 2002, 277:16391-16395.

13. Agris PF: Bringing order to translation: the contributions of transfer RNA anticodon-domain modifications. EMBO Rep 2008, 9:629-635.

14. Murphy FV, Ramakrishnan V, Malkiewicz A, Agris PF: The role of modifications in codon discrimination by tRNA(Lys)UUU. Nat Struct Mol Biol 2004, 11:1186-1191.

15. Hüttenhofer A, Weiss-Brummer B, Dirheimer G, Martin RP: A novel type of +1 frameshift suppressor: a base substitution in the anticodon stem of a yeast mitochondrial serine-tRNA causes frameshift suppression. EMBO J 1990, 9:551-558.

16. Tuohy TM, Thompson S, Gesteland RF, Atkins JF: Seven, eight and ninemembered anticodon loop mutants of tRNA(2Arg) which cause +1 frameshifting. Tolerance of DHU arm and other secondary mutations. J Mol Biol 1992, 228:1042-1054

17. Urbonavicius J, Qian Q, Durand JM, Hagervall TG, Björk GR: Improvement of reading frame maintenance is a common function for several tRNA modifications. EMBO J 2001, 20:4863-4873.

18. Phelps SS, Malkiewicz A, Agris PF, Joseph S: Modified nucleotides in tRNA (Lys) and tRNA(Val) are important for translocation. J Mol Biol 2004, 338:439-444.

19. Sylvers LA, Rogers KC, Shimizu M, Ohtsuka E, Söll D: A 2-thiouridine derivative in tRNAGlu is a positive determinant for aminoacylation by Escherichia coli glutamyl-tRNA synthetase. Biochemistry 1993, 32:3836-3841.

20. Giegé R, Sissler M, Florentz C: Universal rules and idiosyncratic features in tRNA identity. Nucleic Acids Res 1998, 26:5017-5035.

21. Beuning PJ, Musier-Forsyth K: Transfer RNA recognition by aminoacyltRNA synthetases. Biopolymers 1999, 52:1-28.

22. Ibba M, Soll D: Aminoacyl-tRNA synthesis. Annu Rev Biochem 2000, 69:617-650.

23. Safro MG, Moor NA: Codases: fifty years after. Mol Biol (Mosk) 2009, 43:230-242.

24. Crick FH: Codon-anticodon pairing: the wobble hypothesis. J Mol Biol 1966, 19:548-555.

25. Nishimura S: Minor components in transfer RNA: their characterization, location, and function. Prog Nucleic Acid Res Mol Biol 1972, 12:49-85.

26. Sprinzl M, Vassilenko KS: Compilation of tRNA sequences and sequences of tRNA genes. Nucleic Acids Res 2005, 33(Database issue):D139-D140.
27. Agris PF: The importance of being modified: roles of modified nucleosides and $\mathrm{Mg} 2+$ in RNA structure and function. Prog Nucleic Acid Res Mol Biol 1996, 53:79-129.

28. Takai K, Yokoyama S: Roles of 5-substituents of tRNA wobble uridines in the recognition of purine-ending codons. Nucleic Acids Res 2003 31:6383-6391.

29. Wei F-Y, Tomizawa K: Functional loss of Cdkal1, a novel tRNA modification enzyme, causes the development of type 2 diabetes. Endocr J 2011, 58:819-825.

30. Wei F-Y, Suzuki T, Watanabe S, Kimura S, Kaitsuka T, Fujimura A, Matsui $H_{\text {, }}$ Atta M, Michiue H, Fontecave M, Yamagata K, Suzuki T, Tomizawa K: Deficit of tRNA(Lys) modification by Cdkal1 causes the development of type 2 diabetes in mice. J Clin Invest 2011, 121:3598-3608.

31. Wei F-Y, Tomizawa K: Development of type 2 diabetes caused by a deficiency of a tRNAlys modification. Islets 2012, 4:

32. Tseng WC, Medina D, Randerath K: Specific inhibition of transfer RNA methylation and modification in tissues of mice treated with 5-fluorouracil. Cancer Res 1978, 38:1250-1257.

33. Kuchino Y, Borek E, Grunberger D, Mushinski JF, Nishimura S: Changes of post-transcriptional modification of wye base in tumor-specific tRNAPhe. Nucleic Acids Res 1982, 10:6421-6432.

34. Aytaç U, Gündüz U: Q-modification of tRNAs in human brain tumors. Cancer Biochem Biophys 1994, 14:93-98.

35. Pathak C, Jaiswal YK, Vinayak M: Modulation in the activity of lactate dehydrogenase and level of c-Myc and c-Fos by modified base queuine in cancer. Cancer Biol Ther 2008, 7:85-91.

36. Yasukawa T, Suzuki T, Ueda T, Ohta S, Watanabe K: Modification defect at anticodon wobble nucleotide of mitochondrial tRNAs(Leu)(UUR) with pathogenic mutations of mitochondrial myopathy, encephalopathy, lactic acidosis, and stroke-like episodes. J Biol Chem 2000, 275:4251-4257.

37. Isel C, Marquet R, Keith G, Ehresmann C, Ehresmann B: Modified nucleotides of tRNA(3Lys) modulate primer/template loop-loop interaction in the initiation complex of HIV-1 reverse transcription. J Biol Chem 1993, 268:25269-25272.

38. Novoa EM, Pavon-Eternod M, Pan T, Ribas de Pouplana L: A role for tRNA modifications in genome structure and codon usage. Cell 2012, 149:202-213.

39. Czerwoniec A, Dunin-Horkawicz S, Purta E, Kaminska KH, Kasprzak JM, Bujnicki JM, Grosjean H, Rother K: MODOMICS: a database of RNA modification pathways. 2008 update. Nucleic Acids Res 2009, 37(Database issue):D118-D121.

40. Machnicka MA, Milanowska K, Osman Oglou O, Purta E, Kurkowska M, Olchowik A, Januszewski W, Kalinowski S, Dunin-Horkawicz S, Rother KM Helm M, Bujnicki JM, Grosjean H: MODOMICS: a database of RNA modification pathways-2012 update. Nucleic Acids Res 2012, 41:D262-D267.

41. Crooks GE, Hon G, Chandonia J-M, Brenner SE: WebLogo: a sequence logo generator. Genome Res 2004, 14:1188-1190.

42. Ahmed F, Raghava GPS: Designing of highly effective complementary and mismatch siRNAs for silencing a gene. PLOS ONE 2011, 6:e23443.

43. Hofacker IL: Vienna RNA secondary structure server. Nucleic Acids Res 2003, 31:3429-3431.

44. Sato K, Kato Y, Hamada M, Akutsu T, Asai K: IPknot: fast and accurate prediction of RNA secondary structures with pseudoknots using integer programming. Bioinformatics 2011, 27:185-i93.

45. Lowe TM, Eddy SR: tRNAscan-SE: a program for improved detection of transfer RNA genes in genomic sequence. Nucleic Acids Res 1997, 25:955-964.

46. Panwar B, Gupta S, Raghava GP: Prediction of vitamin interacting residues in a vitamin binding protein using evolutionary information. BMC Bioinformatics 2013, 14:44

47. Cochella L, Brunelle JL, Green R: Mutational analysis reveals two independent molecular requirements during transfer RNA selection on the ribosome. Nat Struct Mol Biol 2007, 14:30-36.

48. Panwar B, Raghava GPS: Prediction and classification of aminoacyl tRNA synthetases using PROSITE domains. BMC Genomics 2010, 11:507.

49. Panwar B, Raghava GPS: Predicting sub-cellular localization of tRNA synthetases from their primary structures. Amino Acids 2012, 42:1703-1713.

50. Vapnik VN: An overview of statistical learning theory. IEEE Trans Neural Netw 1999, 10:988-999. 
51. Joachims T: Making large-scale SVM learning practical. Adv Kernel Methods Support Vector Learn 1999, :169-184 [Advances in Kernel Methods - Support Vector Learning].

52. Chou KC, Zhang CT: Prediction of protein structural classes. Crit Rev Biochem Mol Biol 1995, 30:275-349.

53. Panwar B, Arora A, Raghava GPS: Prediction and classification of ncRNAs using structural information. BMC Genomics 2014, 15:127.

54. Kumar R, Panwar B, Chauhan JS, Raghava GP: Analysis and prediction of cancerlectins using evolutionary and domain information. BMC Res Notes 2011, 4:237.

55. Darty K, Denise A, Ponty Y: VARNA: interactive drawing and editing of the RNA secondary structure. Bioinformatics 2009, 25:1974-1975.

doi:10.1186/1471-2105-15-326

Cite this article as: Panwar and Raghava: Prediction of uridine modifications in tRNA sequences. BMC Bioinformatics 2014 15:326.

\section{Submit your next manuscript to BioMed Central and take full advantage of:}

- Convenient online submission

- Thorough peer review

- No space constraints or color figure charges

- Immediate publication on acceptance

- Inclusion in PubMed, CAS, Scopus and Google Scholar

- Research which is freely available for redistribution 